\title{
Comparative analysis between FAR and ARL based control charts with runs rules
}

\author{
Rashid Mehmood ${ }^{1}\left(\mathbb{D}\right.$, Muhammad Hisyam Lee*1 $^{*}$, Iftikhar Ali $^{2}$ (D), Muhammad Riaz $^{3}$ (D) \\ ${ }^{1}$ Department of Mathematical Sciences, Universiti Teknologi Malaysia, Malaysia \\ ${ }^{2}$ Department of Mathematics, University of Hafr Al Batin, Saudi Arabia \\ ${ }^{3}$ Department of Mathematics and Statistics, King Fahad University of Petroleum and Minerals, \\ Saudi Arabia
}

\begin{abstract}
In this study, we have conducted comparative analysis between false alarm rate (FAR) and average run length (ARL) based control charts with runs rules. In this regard, we have considered various univariate and multivariate control charts which include mean, standard deviation, variance, Hotelling, and generalized variance. For evaluation purpose, we have used actual false alarm rate, power, in-control actual average run length, and outof-control average run length as performance indicators. Furthermore, the performance indicators are calculated through Monte Carlo simulation procedures. Results revealed that performance order of runs rules with FAR based control charts are persistent whereas, performance order of runs rules with ARL based control charts are dependent on the circumstances, that is, sample size, size of shift, type of control chart, and side of control limit (upper-sided and lower-sided). Besides, we have provided a real life example using the data on electrical resistance of insulation. In this approach, we have determined that behavior of FAR and ARL based control charts using the real data is recorded similar to the behavior using the statistical performance indicators.
\end{abstract}

Mathematics Subject Classification (2020). 62N05, 62F10, 62F12, 62F15, 62F25, $62 \mathrm{~F} 40$

Keywords. Average run length, control chart, false alarm rate, performance indicators, power, probability of single point, runs rules

\section{Introduction}

The theory of control charts was first proposed by Walter A. Shewhart in 1931 [17] for the detection of assignable causes of variations in a parameter (location and dispersion) of a process characteristics. The assignable causes of variations are unnaturally appeared in an ongoing process, and they are usually occurred due to improper adjustment of controller, operators error, and low quality of batch material. A control chart based on the concept of Shewhart [17] is often known as Shewhart-type control chart. The Shewhart-type control chart based on classical runs rule (any single point out-of-control) is generally considered

\footnotetext{
*Corresponding Author.

Email addresses: mr.rashid_mehmood@yahoo.com (R. Mehmood), mhl@utm.my (M.H. Lee), iftikharali4u@gmail.com (I. Ali),riaz76qau@yahoo.com (M. Riaz)

Received: 27.06.2020; Accepted: 25.10.2020
} 
less efficient for detection of small variations in a parameter [13]. However, to increase the ability of Shewhart control charts towards detection of small variations, Western [21] recommended sensitizing rules or runs rules (also known as decision rules). With passage of time, various authors introduced new forms of sensitizing rules as well as explored their behavior in forms of actual in-control average run length (abbr. as AIARL and denoted as $A R L_{a c t}$ ) and out-of-control average run length (abbr. as OARL and denoted as $\left.A R L_{1}\right)$ such as $[3-6,10,15,18-20]$. The AIARL is an actual value of the average number of sample points that stayed in-control before declaring a process out-of-control on the basis of decision points when in-fact process is in-control. Furthermore, OARL is the average number of sample points that stayed in-control before declaring a process out-of-control on the basis of decision points when actually process is out-of-control.

Champ and Woodall [3] investigated the AIARL as well as OARL of different sensitizing rules. In addition, they used Markov Chain approach as computational technique. Their results showed that although simultaneously implementation of sensitizing rules enhanced the detection ability of Shewhart type control chart but at the same time generated another issue. The issue stated as AIARL deviated from intended level, that is, substantially degraded. To overcome the issue of sensitizing or runs rules, many authors recommended to incorporate the correct value of in-control probability of single point (abbr. as IPSP and denoted as $\left.p_{0}\right)$ into the design structure of Shewhart type control chart $[4,5,8,10,15,22]$. The IPSP is defined as the probability of an out-of-control signal when in-fact a process is in-control. Furthermore, IPSP is generally computed through involving an appropriate method by taking into account an independent choice of runs rules and prefix value of FAR (denoted as $\alpha$ ) or in-control ARL (denoted as $A R L_{0}$ ). The prefix value of $\alpha$ can be defined as the prefix value of probability of decision points for a given choice of runs rule when in-fact a process is in-control. On the other hand, $A R L_{0}$ is the prefix value of the average number of sample points that should be stayed in-control before declaring a process out-of-control on the basis of decision points when in-fact process is in-control.

The appropriate method for computing the IPSP is considered important in designing of Shewhart-type control charts. For instance, Klein [5] computed IPSP based on Markov chain approach for designing and evaluating the mean $(\bar{X})$ control chart. Khoo [4] established graphical plots based on Markov chain approach to obtain the IPSP of existing and proposed runs rules. In addition, he applied the probabilities of single point in the construction of $\bar{X}$ control chart. Shepherd et al. [16] computed the IPSP based on Markov chain approach for designing and evaluation of attribute control chart under runs rules. In continuation, Riaz et al. [15] utilized the proposed equation for designing the FAR based upper-sided mean (symbolized as $\bar{X}_{U}$ ), variance $\left(S_{U}^{2}\right)$, standard deviation $\left(S_{U}\right)$ and range $\left(R_{U}\right)$ control charts. In addition, they showed that proposed equations play its role to maintain the AFAR of FAR based $\bar{X}_{U}, S_{U}^{2}, S_{U}$ and $R_{U}$ control charts under runs rules at $\alpha$. The applications of polynomial equation by [15] can be seen in various studies such as $[9,11,12,22]$. In this particular research direction, Mehmood et al. [8] offered new polynomial equation alternative to the study by [15] for increasing the detection ability of two sided Shewhart-type control chart under runs rules.

The aforementioned literature review is representing the FAR and ARL based control charts. A control chart depends on the $\alpha$ is termed as FAR based control chart such as $[15,22]$. Likewise, ARL based control chart depends on the $A R L_{0}$ such as $[4,5]$. It is valuable to mention that numbers of studies have been seen on the topic of FAR and ARL based control charts separately. In this research direction, it is very rare to find study on the comparative analysis between FAR and ARL based control charts. This has taken as the motivation of current study.

This study aims to conduct comparative analysis between FAR and ARL based control charts with runs rules. To achieve the goal, we will construct design structures of upper-sided and lower-sided univariate and multivariate control charts with runs rules. 
The upper-sided and lower-sided univariate control charts include mean $\left(\bar{X}_{U}\right.$ and $\left.\bar{X}_{L}\right)$, variance $\left(S_{U}^{2}\right.$ and $\left.S_{L}^{2}\right)$, and standard deviation $\left(S_{U}\right.$ and $\left.S_{L}\right)$. Furthermore, upper-sided and lower-sided multivariate control charts contain generalized variance $\left(|S|_{U}\right.$, and $\left.|S|_{L}\right)$ and Hotelling's $\left(T_{U}^{2}\right)$. Besides, we will evaluate the performance of FAR and ARL based control charts by considering the AFAR, power (denoted as $P_{1}$ ), AIARL, and OARL as performance measures. The $P_{1}$ is defined as the probability of the decision points for a given choice of runs rule that are declared out-of-control when in-fact the process is out-of-control. In addition, for computation of the performance measures, we will illustrate and also employ the Monte Carlo simulation procedures without loss of generality. Furthermore, we will conduct comparative analysis on the behavior of FAR and ARL based control charts under classical and additional runs rules. All of the prescribed methods for comparative analysis cover the statistical aspects of current study. To highlight the practical significance of the study, a real life example will be presented using the data on electrical resistance of insulation.

Rest of the article is organized as follows: In Section 2, we will construct different design structures of FAR and ARL based control charts with classical and additional runs rules. In Section 3, we will discuss Monte Carlo simulation procedure for computing different performance measures of each control chart under consideration, and also conduct comparative analysis. In Section 4, we will give a real life example using the data on electrical resistance of insulation to compare the behavior of FAR and ARL based control charts with runs rules. Lastly, we will summarize and conclude the whole study in Section 5 .

\section{Design structures of FAR and ARL based Shewhart-type control charts under runs rules}

In this section, we construct FAR and ARL based design structures of the Shewharttype control charts under runs rules. Now assume that a process characteristic $X$ follows a normal distribution and characteristics $\left(Y_{1}, Y_{2}\right)$ follow bivariate normal distribution.

\section{1. $\bar{X}_{U}$ control chart}

Let $\bar{X}_{j}, j=1,2,3, \ldots$ denote the $j$ th plotting statistic of sample of size $n$. Thus, a process is said be out-of-control if $k / k$ or $k / k+r$ consecutive statistic $\bar{X}_{j}$ falling above the control limit $U_{\bar{X}}$. The $\bar{X}_{j}$ and $U_{\bar{X}}$ are formulated as follows:

$$
\bar{X}_{j}=\frac{1}{n} \sum_{i=1}^{n} X_{i j}, \quad U_{\bar{X}}=\mu_{0}+Z_{\left(1-p_{0}\right)} \frac{\sigma_{0}}{\sqrt{n}},
$$

where $\mu_{0}$ and $\sigma_{0}$ are known in-control mean and standard deviation of $X, Z_{\left(1-p_{0}\right)}$ is $\left(1-p_{0}\right)$ th percentile of standard normal distribution [13]. Furthermore, choice of $p_{0}$ depends on the prefix value of $k / k$ or $k / k+r$ runs rules and $\alpha$ or $A R L_{0}$. The correct value of $p_{0}$ is desired to sustain the $\alpha_{a c t}$ or $A R L_{a c t}$ of a control chart at $\alpha$ or $A R L_{0}$, respectively. To compute the required $p_{0}$ value, one of the best solutions provided by [15] in the form of a polynomial equation for handling the FAR based control charts. Riaz et al. [15] introduced exact polynomial equation for computing the required $p_{0}$ value as per the given choice of $k / k$ or $k / k+r$ and $\alpha$. Thus, polynomial equation for computing the $p_{0}$ as per the given choice of $k / k$ and $\alpha$ or $A R L_{0}$ are given as:

$$
\begin{cases}p_{0}=\sqrt[k]{\alpha}, & \text { if } \alpha \text { is given, } \\ A R L_{0}\left(1-p_{0}\right) p_{0}^{k}+p_{0}^{k}-1=0, & \text { if } A R L_{0} \text { is given. }\end{cases}
$$


To cover the case of $k$ out of $k+r$ (denoted as $k / k+r, r \geq 1$ ) runs rules, expressions to obtain $p_{0}$ for the given value of $\alpha$ or $A R L_{0}$ are as follows:

$$
\begin{cases}\alpha=\left(\begin{array}{c}
k+r \\
k
\end{array}\right) p_{0}^{k}\left(1-p_{0}\right)^{r}, & \text { if } \alpha \text { is given, } \\
p_{0}=R\left(k \mid k+r, A R L_{0}\right), & \text { if } A R L_{0} \text { is given, }\end{cases}
$$

where $R\left(k \mid k+r, A R L_{0}\right)$ denote a constant, lies between zero and one, and it depends on the given value of $k / k+r$ and $A R L_{0}$. Besides, a control chart dependent on $\alpha$ is termed as FAR based control chart. Similarly, a control chart contingent on $A R L_{0}$ is called ARL based control chart. The theoretical justification of Eqs.(2.1)-(2.2) when $\alpha$ given can be seen in [8]. In addition, theoretical illustration of Eq.(2.1) when $A R L_{0}$ given is as follows: The probability distribution (also called run length distribution) of $k / k$ consecutive statistics breached the control limit is generalized geometric distribution of order $k$ with parameter $p_{0}$ [2]. As our interest is to find out correct value of $p_{0}$ so that $A R L_{a c t}$ of a Shewhart-type control remains equal to $A R L_{0}$. Therefore, we equate the mean of generalized geometric distribution of order $k$ with parameter $p_{0}$ to $A R L_{0}$. Note that value of $p_{0}$ in Eq. (2.2) when $A R L_{0}$ given is hard to obtain by analytical approach. However, one may calculate using a computational technique (e.g. Monte Carlo simulation) with a condition that $A R L_{\text {act }}$ remains equal to $A R L_{0}$.

\section{2. $\bar{X}_{L}$ control chart}

Let $\bar{X}_{j}, j=1,2,3, \ldots$ denote the $j$ th plotting statistic of sample of size $n$. Thus, a process is said be out-of-control if $k / k$ or $k / k+r$ consecutive $\bar{X}_{j}$ falling below the $L_{\bar{X}}$. The $\bar{X}_{j}$ and $L_{\bar{X}}$ are formulated as follows:

$$
\bar{X}_{j}=\frac{1}{n} \sum_{i=1}^{n} X_{i j}, \quad L_{\bar{X}}=\mu_{0}+Z_{p_{0}} \frac{\sigma_{0}}{\sqrt{n}},
$$

where $Z_{p_{0}}$ is $p_{0}$ th percentiles of standard normal distribution [13]. Rest of the discussion remained similar to Section 2.1.

\section{3. $S_{U}^{2}$ and $S_{L}^{2}$ control charts}

Let $S_{j}^{2}, j=1,2,3, \ldots$ denote the $j$ th plotting statistic of sample of size $n$. Thus, a process is said be out-of-control if $k / k$ or $k / k+r$ consecutive $S_{j}^{2}$ crossed the control limit $\left(U_{S^{2}}\right.$ for $S_{U}^{2}$ or $L_{S^{2}}$ for $S_{L}^{2}$ control chart). The $S_{j}^{2}, U_{S^{2}}$, and $L_{S^{2}}$ are formulated as follows:

$$
S_{j}^{2}=\frac{1}{n-1} \sum_{i=1}^{n}\left(X_{i j}-\bar{X}_{j}\right), \quad U_{S^{2}}=\frac{w_{U} \sigma_{0}^{2}}{n-1}, \quad L_{S^{2}}=\frac{w_{L} \sigma_{0}^{2}}{n-1},
$$

where $w_{U}$ and $w_{L}$ are $\left(1-p_{0}\right)$ th and $p_{0}$ th percentiles of chi-squared distribution with $n-1$ degree of freedom, and $\sigma_{0}^{2}$ is known in-control variance of $X$.

\section{4. $S_{U}$ and $S_{L}$ control charts}

Let $S_{j}, j=1,2,3, \ldots$ denote the $j$ th plotting statistic of sample of size $n$. Thus, a process is said be out-of-control if $k / k$ or $k / k+r$ consecutive $S_{j}$ falls outside the control limit $\left(U_{S}\right.$ for $S_{U}$ or $L_{S}$ for $S_{L}$ control chart). The $S_{j}, U_{S}$, and $L_{S}$ are formulated as follows:

$$
S_{j}=\sqrt{\frac{\sum_{i=1}^{n}\left(X_{i j}-\bar{X}_{j}\right)}{n-1}}, \quad U_{S}=\frac{m_{U} \sigma_{0}}{\sqrt{n-1}}, \quad L_{S}=\frac{m_{L} \sigma_{0}}{\sqrt{n-1}},
$$

where $m_{U}$ and $m_{L}$ are $\left(1-p_{0}\right)$ th and $p_{0}$ th percentiles of chi distribution with $n-1$ degree of freedom, and $\sigma_{0}$ is known in-control standard deviation of $X$. 


\subsection{Bivariate $T_{U}^{2}$ control chart}

Let $T_{j}^{2}, j=1,2,3, \ldots$ denote the $j$ th plotting statistic of sample of size $n$. Thus, a process is said be out-of-control if $k / k$ or $k / k+r$ consecutive $T_{j}^{2}$ lies beyond the $U_{T^{2}}$. The $T_{j}^{2}$ and $U_{T^{2}}$ are formulated as follows:

$$
T_{j}^{2}=n\left(M_{j}-\underline{\mu_{0}}\right)^{t} \Sigma_{0}^{-1}\left(M_{j}-\underline{\mu_{0}}\right), \quad U_{T^{2}}=t_{U}^{2},
$$

where $M_{j}=\left(\bar{Y}_{1 j}, \bar{Y}_{2 j}\right)^{t}$ is the $j$ th sample mean vector, $\mu_{0}=\left(\mu_{10}, \mu_{20}\right)^{t}$ is known in-control mean vector of $Y_{1}$ and $Y_{2}, \Sigma_{0}$ is variance-covariance matrix of $M_{j}$, and $t_{U}^{2}$ is $\left(1-p_{0}\right)$ th percentile of chi-squared distribution with two degree of freedom.

\subsection{Bivariate $|S|_{U}$ and $|S|_{L}$ control charts}

Let $|S|_{j}, j=1,2,3, \ldots$ denote the $j$ th plotting statistic of sample of size $n$. Thus, a process is said be out-of-control if $k / k$ or $k / k+r$ consecutive $|S|_{j}$ falls outside the control limit $\left(U_{|S|}\right.$ for $|S|_{U}$ or $L_{|S|}$ for $|S|_{L}$ control chart). The $S_{j}, U_{S}$, and $L_{S}$ are formulated as follows:

$$
|S|_{j}=S_{1 j}^{2} S_{2 j}^{2}-S_{12 j}^{2}, \quad U_{|S|}=\frac{\left|\Sigma_{0}\right| b_{U}^{2}}{4(n-1)^{2}}, \quad L_{|S|}=\frac{\left|\Sigma_{0}\right| b_{L}^{2}}{4(n-1)^{2}},
$$

where $S_{1 j}^{2}$ and $S_{2 j}^{2}$ are $j$ th sample variance of size $n, S_{12 j}^{2}$ is sample covariance between process characteristics $\left(Y_{1}\right.$ and $\left.Y_{2}\right), b_{U}$ and $b_{L}$ are $\left(1-p_{0}\right)$ th and $p_{0}$ th percentiles of chisquared distribution with $2 n-4$ degree of freedom, and $\left|\Sigma_{0}\right|$ is the determinants of $\Sigma_{0}$.

\section{Computation of performance measures and comparative analysis}

In this section we are intended to provide Monte Carlo simulation procedure $[7,15]$ for computing the performance measures of upper-sided and lower-sided control charts under runs rules (see Sec. 2), and also conduct comparative analysis. The performance measures are $\alpha_{a c t}, P_{1}, A R L_{a c t}$, and $A R L_{1}$, and their further details are given in Sec. 1. A control chart for different choices of runs rules is said to be best if $\alpha_{a c t}$ or $A R L_{a c t}$ is equal to $\alpha$ or $A R L_{o}$, respectively. Likewise, a control chart under different choices of runs rules can be announced best for a certain choice of runs rule if it attains minimum $A R L_{1}$ or maximum $P_{1}$ given that the control chart has same $A R L_{0}$ or $\alpha$ respectively.

\section{1. $\bar{X}_{U}$ and $\bar{X}_{L}$ control charts}

To compute the $P_{1}$ of $\bar{X}_{U}$ control chart, generate $10^{5}$ random samples of size $n$ from normal distribution with out-of-control mean $\mu^{*}=\mu_{0}+\delta_{1} \sigma_{0}$ (where $\delta_{1} \geq 0$ represents amount of upward shift) and in-control standard deviation $\sigma_{0}$ followed by calculating the plotting statistics $\left(\bar{X}_{j}\right)$ and comparing them with $U_{\bar{X}}$ to count the number of statistics falling above the $U_{\bar{X}}$. Finally, proportion of plotting statistics falling above the $U_{\bar{X}}$ is reported as $P_{1}$. Similarly, one may proceed for $\bar{X}_{L}$ control chart by considering $L_{\bar{X}}$ with $\mu^{*}=\mu_{0}+\delta_{2} \sigma_{0}$ (where $\delta_{2} \leq 0$ represents amount of downward shift). Furthermore, for computing the $A R L_{1}$, generate a random sample of size $n$ from normal distribution followed by calculating the statistics to compare with the $U_{\bar{X}}$ or $L_{\bar{X}}$ for deciding either process is in-control or out-of-control. Afterwards, repeat the prescribed procedure until the process is declared out-of-control and then record the sample number (run length). Likewise, repeat the aforementioned procedure $10^{5}$ times to attain the vector of run length. Ultimately, average of the vector of run length is required $A R L_{1}$. Note that $\alpha_{a c t}$ and $A R L_{a c t}$ is the special case of $P_{1}$ and $A R L_{1}$, respectively when $\delta_{1}=\delta_{2}=0$. Based on the aforesaid procedures, we have attained $\alpha_{a c t}, A R L_{a c t}, P_{1}$ and $A R L_{1}$ of $\bar{X}_{U}$ and $\bar{X}_{L}$ control charts for some selective choices of $\delta_{1}, \delta_{2}, n, \alpha=0.0027, A R L_{0}=370, k / k$ and $k / k+r$ (see Tables 1-3). Thus, the results are discussed as follows: 
- The $\alpha_{a c t}$ and $A R L_{0}$ of mean control charts $\left(\bar{X}_{U}\right.$ and $\left.\bar{X}_{L}\right)$ are obtained equal to $\alpha$ and $A R L_{0}$ (i.e. $\alpha_{a c t}=\alpha=0.0027$ and $\left.A R L_{a c t}=A R L_{0}=370\right)$ for classical and additional runs rules (see Table 1). This means that Eqs.(2.1)-(2.2) plays its role for resolving the issue of Shewhart-type control charts under runs rules. The details about the issue of Shewhart-type control charts are given in Sec. 1.

- Behavior of FAR based mean control charts with runs rules are sustained in terms of $P_{1}$ (see Tables 2-3). Similarly, we have observed for the case of ARL based mean control charts in terms of $A R L_{1}$. These outcomes can be interpreted as detection ability of $\bar{X}_{U}$ control chart is similar to the $\bar{X}_{L}$ control chart when in-control process mean is shifted to new level with same magnitude of distance.

- The detection ability of FAR based mean control charts are observed uniformly higher for all choices of shifts $\left(\delta_{1}>0\right.$ and $\left.\delta_{2}<0\right)$ in terms of $P_{1}$ when additional runs rules are employed as compared to $1 / 1$ runs rule (see Tables $2-3$ ). In continuation, detection ability of ARL based mean control charts are found higher for only small-to-moderate shifts (e.g. $0<\delta_{1}<1$ ) in terms of $A R L_{1}$ when additional runs rules are implemented relative to classical runs rule. This implies that ARL based mean control charts are efficient towards detection of small-to-moderate shifts when additional runs rules are considered, and also efficient for large shifts when classical runs rule is incorporated.

- There are relationships between detection ability and choices of $k / k, k / k+r, n$, $\delta_{1}$ and $\delta_{2}$ (see Tables 2-3). For instance, detection ability of FAR based mean control charts uniformly increase as value of $k / k$ increases. This remains valid for all choices of $n, \delta_{1}$ and $\delta_{2}$. Also, detection ability of ARL based mean control charts increase as value of $k / k$ increases.

- Among variant choices of runs rules, the $3 / 4$ with mean control charts is proved efficient towards detection of small-to-moderate shifts relative to the other choices. Also, based on the detection ability in terms of $A R L_{1}$ and $P_{1}$, performance order of runs rules with mean control charts is $3 / 4,3 / 3,2 / 4,2 / 2,2 / 3$, and $1 / 1$.

Table 1. $\alpha_{a c t}$ and $A R L_{a c t}$ at $\alpha=0.0027, A R L_{0}=370, \delta_{1}=0, \delta_{2}=0, \delta_{3}=1$, $\delta_{4}=1, d^{*}=1, d=0, k / k$ and $k / k+r$

\begin{tabular}{|c|c|c|c|c|c|c|c|c|c|c|c|c|}
\hline & $1 / 1$ & & $2 / 2$ & & $3 / 3$ & & $2 / 3$ & & $2 / 4$ & & $3 / 4$ & \\
\hline & $\alpha_{a c t}$ & $A R L_{a c t}$ & $\alpha_{a c t}$ & $A R L_{a c t}$ & $\alpha_{a c t}$ & $A R L_{a c t}$ & $\alpha_{a c t}$ & $A R L_{a c t}$ & $\alpha_{a c t}$ & $A R L_{a c t}$ & $\alpha_{a c t}$ & $A R L_{a c t}$ \\
\hline$X_{U}$ & 0.0027 & 370.37 & 0.0027 & 370.17 & 0.0027 & 370.14 & 0.0027 & 370.18 & 0.0027 & 370.10 & 0.0027 & 370.15 \\
\hline $\bar{X}_{L}$ & 0.0027 & 370.37 & 0027 & 70.17 & 0.0027 & 70.13 & 0.0027 & 370.43 & 0.0027 & 0.53 & 0.0027 & 370.10 \\
\hline$S_{U}^{2}$ & 0.0027 & 370.17 & 0.0027 & 370.60 & 0.0027 & 370.40 & 0.0027 & 370.63 & 0.0027 & 370.13 & 0.0027 & 370.72 \\
\hline$S_{L}^{2}$ & 0.0027 & 370.17 & 0.0027 & 370.17 & 0.0027 & 370.14 & 0.0027 & 370.18 & 0.0027 & 370.10 & 0.0027 & 370.43 \\
\hline$S_{U}$ & 0.0027 & 370.23 & 0.0027 & 370.31 & 0.0027 & 370.13 & 0.0027 & 370.43 & 0.0027 & 370.53 & 0.0027 & 371.20 \\
\hline$S_{L}$ & 0.0027 & 370.21 & 0.0027 & 370.28 & 0.0027 & 370.40 & 0.0027 & 370.63 & 0.0027 & 370.13 & 369.71 & 372.42 \\
\hline$|S|_{U}$ & 0.0027 & 370.25 & 0.0027 & 370.17 & 0.0027 & 370.14 & 0.0027 & 370.18 & 0.0027 & 370.10 & 0.0027 & 371.31 \\
\hline$|S|_{L}$ & 0.0027 & 370.37 & 0.0027 & 370.17 & 0.0027 & 370.13 & 0.0027 & 370.43 & 0.0027 & 370.41 & 0.0027 & 372.31 \\
\hline$T_{U}^{2}$ & 0.0027 & 370.37 & 0.0027 & 370.11 & 0.0027 & 370.40 & 0.0027 & 370.63 & 0.0027 & 370.20 & 0.0028 & 371.25 \\
\hline
\end{tabular}

\section{2. $S_{U}^{2}, S_{L}^{2}, S_{U}, S_{L},|S|_{L}$ and $|S|_{U}$ control charts}

The mechanism for computing $P_{1}$ and $A R L_{1}$ of $S_{L}^{2}$ and $S_{U}^{2}$ control charts is similar to $\bar{X}_{L}$ and $\bar{X}_{U}$ control charts except in-control mean is stable $\mu_{0}$, whereas in-control variance $\sigma_{0}^{2}$ is out-of-control, that is, $\sigma_{1}^{2}=\left(\delta_{3} \sigma_{0}\right)^{2}$ and $\sigma_{1}^{2}=\left(\delta_{4} \sigma_{0}\right)^{2}$, where $\delta_{3} \geq 1$ and $\delta_{4} \leq 1$ are upward and downward shift. Likewise, for $S_{U}$ and $S_{L}$ control charts, assume that the in-control mean is stable, whereas standard deviation is out-of-control $\sigma_{1}=\delta_{3} \sigma_{0}$ and $\sigma_{1}=$ $\delta_{4} \sigma_{0}$. Besides, procedures for computing the power and out-of-control average run length of $|S|_{L}$ and $|S|_{U}$ control charts is to assume the $\underline{\mu_{0}}$ is stable, whereas $\Sigma_{0}$ is out-of-control 
Table 2. $P_{1}$ and $A R L_{1}$ of $\bar{X}_{U}$ control chart at $n=5, \alpha=0.0027, A R L_{0}=370$, $k / k, k / k+r$ and $\delta_{1}$

\begin{tabular}{lllllllllllll}
\hline \multicolumn{10}{c}{$2 / 2$} & \multicolumn{1}{c}{$\bar{X}_{U}$} \\
\hline & $1 / 1$ & & $2 / 3$ & & $2 / 3$ & & $2 / 4$ & & $3 / 4$ \\
$\delta_{1}$ & $P_{1}$ & $A R L_{1}$ & $P_{1}$ & $A R L_{1}$ & $P_{1}$ & $A R L_{1}$ & $P_{1}$ & $A R L_{1}$ & $P_{1}$ & $A R L_{1}$ & $P_{1}$ & $A R L_{1}$ \\
\hline 0 & 0.0027 & 370.37 & 0.0027 & 370.76 & 0.0027 & 370.03 & 0.0026 & 372.02 & 0.0028 & 371.70 & 0.0027 & 369.47 \\
0.05 & 0.0038 & 263.16 & 0.0042 & 240.47 & 0.0045 & 228.97 & 0.0043 & 243.52 & 0.0047 & 233.21 & 0.0048 & 225.25 \\
0.1 & 0.0053 & 188.68 & 0.0065 & 159.79 & 0.0074 & 146.52 & 0.0069 & 155.64 & 0.0077 & 151.97 & 0.0082 & 136.01 \\
0.15 & 0.0072 & 138.89 & 0.0097 & 108.75 & 0.0117 & 96.92 & 0.0107 & 106.06 & 0.0122 & 102.20 & 0.0136 & 89.33 \\
0.2 & 0.0098 & 102.04 & 0.0142 & 75.73 & 0.018 & 66.16 & 0.0164 & 71.05 & 0.019 & 69.54 & 0.0219 & 59.05 \\
0.25 & 0.0131 & 76.34 & 0.0204 & 53.9 & 0.027 & 46.6 & 0.0244 & 50.62 & 0.0288 & 48.99 & 0.034 & 41.87 \\
0.3 & 0.0174 & 57.47 & 0.0288 & 39.23 & 0.0392 & 33.84 & 0.0355 & 36.90 & 0.0426 & 34.92 & 0.0513 & 29.75 \\
0.35 & 0.0228 & 43.86 & 0.0398 & 29.19 & 0.0556 & 25.28 & 0.0505 & 27.64 & 0.0615 & 26.00 & 0.0747 & 21.88 \\
0.4 & 0.0295 & 33.9 & 0.0539 & 22.19 & 0.0767 & 19.41 & 0.0702 & 20.55 & 0.0863 & 19.74 & 0.1056 & 17.12 \\
1 & 0.2925 & 3.42 & 0.5316 & 3.22 & 0.6708 & 3.88 & 0.7029 & 3.11 & 0.8065 & 3.14 & 0.8406 & 3.611 \\
\hline
\end{tabular}

Table 3. $P_{1}$ and $A R L_{1}$ of $\bar{X}_{L}$ control chart at $n=5, \alpha=0.0027, A R L_{0}=370$, $k / k, k / k+r$ and $\delta_{2}$

\begin{tabular}{|c|c|c|c|c|c|c|c|c|c|c|c|c|}
\hline \multicolumn{13}{|c|}{$\bar{X}_{L}$} \\
\hline & $1 / 1$ & & $2 / 2$ & & $3 / 3$ & & $2 / 3$ & & $2 / 4$ & & $3 / 4$ & \\
\hline$\delta_{2}$ & $P_{1}$ & $A R L_{1}$ & $P_{1}$ & $A R L_{1}$ & $P_{1}$ & $A R L_{1}$ & $P_{1}$ & $A R L_{1}$ & $P_{1}$ & $A R L_{1}$ & $P_{1}$ & $A R L_{1}$ \\
\hline 0 & 0.0027 & 370.37 & 0.0027 & 370.76 & 0.0027 & 370.03 & 0.0026 & 370.15 & 0.0028 & 369.27 & 0.0027 & 367.20 \\
\hline-0.05 & 0.0038 & 263.16 & 0.0042 & 240.47 & 0.0045 & 228.97 & 0.0043 & 239.73 & 0.0047 & 232.81 & 0.0048 & 219.60 \\
\hline-0.1 & 0.0053 & 188.68 & 0.0065 & 159.79 & 0.0074 & 146.52 & 0.0069 & 158.33 & 0.0077 & 148.88 & 0.0082 & 138.42 \\
\hline-0.15 & 0.0072 & 138.89 & 0.0097 & 108.75 & 0.0117 & 96.92 & 0.0107 & 106.96 & 0.0122 & 101.41 & 0.0136 & 89.84 \\
\hline-0.2 & 0.0098 & 102.04 & 0.0142 & 75.73 & 0.018 & 66.16 & 0.0164 & 71.94 & 0.019 & 68.35 & 0.0219 & 59.87 \\
\hline-0.25 & 0.0131 & 76.34 & 0.0204 & 53.9 & 0.027 & 46.6 & 0.0244 & 51.56 & 0.0288 & 48.12 & 0.034 & 42.35 \\
\hline-0.3 & 0.0174 & 57.47 & 0.0288 & 39.23 & 0.0392 & 33.84 & 0.0355 & 37.01 & 0.0426 & 34.98 & 0.0513 & 30.04 \\
\hline-0.35 & 0.0228 & 43.86 & 0.0398 & 29.19 & 0.0556 & 25.28 & 0.0505 & 27.25 & 0.0615 & 26.00 & 0.0747 & 22.00 \\
\hline-0.4 & 0.0295 & 33.9 & 0.0539 & 22.19 & 0.0767 & 19.41 & 0.0702 & 20.38 & 0.0863 & 20.11 & 0.1056 & 16.98 \\
\hline-1 & 0.2925 & 3.42 & 0.5316 & 3.22 & 0.6708 & 3.88 & 0.7029 & 3.1292 & 0.8065 & 3.12 & 0.8406 & 3.60 \\
\hline
\end{tabular}

$\left(\right.$ say $\left.\Sigma_{1}\right)$, that is,

$$
\underline{\mu_{0}}=\left[\begin{array}{c}
\mu_{10} \\
\mu_{20}
\end{array}\right] \quad \text { and } \quad \Sigma_{1}=\left[\begin{array}{cc}
\delta_{5}^{2} \sigma_{10}^{2} & \delta_{5} \delta_{6} \rho \sigma_{10} \sigma_{20} \\
\delta_{5} \delta_{6} \rho \sigma_{10} \sigma_{20} & \delta_{6}^{2} \sigma_{20}^{2}
\end{array}\right],
$$

where $\delta_{5}^{2} \geq 1$ and $\delta_{6}^{2} \geq 1$ are amount of shifts in the in-control variances $\left(\sigma_{10}^{2}\right.$ and $\left.\sigma_{20}^{2}\right)$, $\rho$ is the amount of correlation between $Y_{1}$ and $Y_{2}$. After that, generate random sample from bivariate normal distribution with $\mu_{0}$ and $\Sigma_{1}$ followed by calculating the $|S|_{U}$ and comparing with the control limit $\left(U_{|S|}\right.$ or $\left.\bar{L}_{|S|}\right)$ to decide whether the process is in-control or out-of-control. Rest of the steps for computing $P_{1}$ and $A R L_{1}$ of $|S|_{L}$ and $|S|_{U}$ control charts are identical to $\bar{X}_{L}$ and $\bar{X}_{U}$ control charts. It is worthy to mention that detection ability of $|S|_{L}$ and $|S|_{U}$ control charts are dependent on the product of shifts $d^{* 2}=\delta_{5}^{2} \delta_{6}^{2}$ and $n$ in respective of the choice of other quantities such as $\mu_{0}$, and $\Sigma_{1}$. This property is termed as invariance property. Therefore, one may consider the product value of shift instead of assuming each shift separately. For comparative purpose, we have obtained $\alpha_{a c t}, A R L_{a c t}$, $P_{1}$ and $A R L_{1}$ of $S_{U}^{2}, S_{L}^{2}, S_{U}, S_{L},|S|_{L}$ and $|S|_{U}$ control charts at $\alpha=0.0027, A R L_{0}=370$, various choices of $k / k, k / k+r, \delta_{3}, \delta_{4}$ and $d^{*}$ (see Tables 4-9). Note that $\alpha_{\text {act }}$ and $A R L_{\text {act }}$ is the special case of $P_{1}$ and $A R L_{1}$, respectively when $\delta_{3}=\delta_{4}=d^{*}=1$. Now discussions on the behavior of $S_{U}^{2}, S_{L}^{2}, S_{U}, S_{L},|S|_{L}$ and $|S|_{U}$ control charts are given in the following points:

- The $\alpha_{a c t}$ and $A R L_{1}$ of $S_{U}^{2}, S_{L}^{2}, S_{U}, S_{L},|S|_{L}$ and $|S|_{U}$ control charts are obtained equal to prefix values of $\alpha$ and $A R L_{0}$ (i.e. $\alpha_{\text {act }}=\alpha=0.0027$ and $A R L_{a c t}=A R L_{0}$ $=370$ ) for classical and additional runs rules (see Table 1). 
- The detection ability of FAR based $S_{U}^{2}$ and $S_{U}$ control charts uniformly increases for small $n$ (e.g. $n<5$ ) in terms of $P_{1}$ as value of $k / k$ increases. In comparison, detection ability of ARL based $S_{U}^{2}$ and $S_{U}$ control charts decreases for small $n$ in terms of $A R L_{1}$ as value of $k / k$ increases. This may illustrate as the $k / k$ runs rules are useful for FAR based $S_{U}^{2}$ and $S_{U}$ control charts at any choice of $n$ relative to $1 / 1$ runs rule, whereas $k / k$ runs rules are not beneficial for ARL based $S_{U}^{2}$ and $S_{U}$ control charts when $n$ is small. However, for $n \geq 5$, detection ability of ARL based $S_{U}^{2}$ and $S_{U}$ control charts with $2 / 2$ and $3 / 3$ runs rules are seen higher at wide range of shifts relative to $1 / 1$ runs rule (see Tables $4 \& 6$ ). Between runs rules, $2 / 2$ results in higher detection ability of ARL based $S_{U}^{2}$ and $S_{U}$ control charts as compared to $3 / 3$.

- The diagnosing ability of FAR based $|S|_{U}$ control chart uniformly increases for small $n$ (e.g. $n<5)$ in terms of $P_{1}$ as value of $k / k$ increases. In contrast, detection ability of ARL based $|S|_{U}$ control chart reduces for small $n$ (e.g. $n<5$ ) in terms of $A R L_{1}$ as $k / k$ increases. This may illustrate as $k / k$ runs rules are useful for FAR based $|S|_{U}$ control chart relative to $1 / 1$ runs rule at any choice of $n$, whereas $k / k$ runs rules are not useful for ARL based $|S|_{U}$ control chart when $n$ is small. However, for $n \geq 5$, detection ability of ARL based $|S|_{U}$ control chart under $k / k$ runs rules is seen higher than $1 / 1$ runs rule (see Table 8 ) at various choices of shifts $\left(1<d^{*}<1.50\right)$. Among $k / k$ runs rules, $3 / 3$ results in highest detection ability of ARL based $|S|_{U}$ control chart for $1<d^{*} \leq 1.20$ relative to $2 / 2$. Similarly, $2 / 2$ results into highest detection ability of ARL based $|S|_{U}$ control chart for $1.20<d^{*} \leq 2.5$ relative to $3 / 3$.

- The detection ability of FAR based $S_{L}^{2}, S_{L}$, and $|S|_{L}$ control charts are uniformly higher when additional runs rules are applied relative to classical runs rule (see Tables $5,7 \& 9$ ). Similarly, detection ability of ARL based $S_{L}^{2}, S_{L}$, and $|S|_{L}$ control charts are observed maximum for small-to-moderate shifts when additional runs rules are employed.

- The $n, \delta_{3}, \delta_{4}$ and $d^{*}$ have an effect on the detection ability of $S_{U}^{2}, S_{L}^{2}, S_{U}, S_{L},|S|_{L}$ and $|S|_{U}$ control charts. In simple words, detection ability of FAR and ARL based control charts increases in terms of $P_{1}$ and $A R L_{1}$ as size of $n, \delta_{3}, \delta_{4}$ and/or $d^{*}$ increases (see Tables 4-9).

- At several choices of small-to-moderate shifts $\left(\delta_{3}, \delta_{4}\right.$ and $\left.d^{*}\right)$, either $2 / 4$ or $3 / 4$ runs rule is proved efficient with dispersion control charts relative to $k / k$ runs rules in general. In terms of $A R L_{1}$ and $P_{1}$, performance order of various runs rules with dispersion control charts is as follows: $2 / 4,3 / 4,2 / 3,2 / 2,3 / 3,1 / 1$ when $S_{U}^{2}$ and $S_{U}$; $3 / 43 / 3,2 / 2,2 / 3$ or $2 / 4,1 / 1$ when $S_{L}^{2}$ and $S_{L}$. Also, for $|S|_{U}$ and $|S|_{L}$ control charts, pattern of various runs rules are almost similar to $S_{U}^{2}$ and $S_{L}^{2}$ control charts.

Table 4. $P_{1}$ and $A R L_{1}$ of $S_{U}^{2}$ control chart at $n=5, \alpha=0.0027, A R L_{0}=370$, $k / k, k / k+r$ and $\delta_{3}$

\begin{tabular}{lllllllllllll}
\hline \multicolumn{10}{c}{$S_{U}^{2}$} \\
\hline & $1 / 1$ & & $2 / 2$ & & $3 / 3$ & & $2 / 3$ & & $2 / 4$ & & $3 / 4$ \\
$n_{3}$ & $P_{1}$ & $A R L_{1}$ & $P_{1}$ & $A R L_{1}$ & $P_{1}$ & $A R L_{1}$ & $P_{1}$ & $A R L_{1}$ & $P_{1}$ & $A R L_{1}$ & $P_{1}$ & $A R L_{1}$ \\
\hline 1 & 0.0027 & 370.37 & 0.0027 & 370.76 & 0.0027 & 370.02 & 0.0026 & 370.30 & 0.0028 & 369.33 & 0.0027 & 368.29 \\
1.10 & 0.0096 & 104.13 & 0.0104 & 101.86 & 0.0109 & 103.72 & 0.0125 & 96.65 & 0.0149 & 88.38 & 0.0137 & 92.38 \\
1.21 & 0.0255 & 39.261 & 0.029 & 39.24 & 0.0313 & 41.48 & 0.0398 & 34.36 & 0.0509 & 32.29 & 0.0452 & 34.14 \\
1.32 & 0.0542 & 18.44 & 0.0632 & 19.36 & 0.0692 & 21.39 & 0.0946 & 17.09 & 0.1256 & 15.56 & 0.1086 & 17.26 \\
1.44 & 0.0977 & 10.23 & 0.115 & 11.44 & 0.1263 & 13.21 & 0.1802 & 10.00 & 0.2417 & 9.42 & 0.2056 & 10.54 \\
1.56 & 0.1552 & 6.44 & 0.1824 & 7.71 & 0.1998 & 9.28 & 0.2901 & 6.77 & 0.3853 & 6.50 & 0.3265 & 7.50 \\
1.69 & 0.2235 & 4.47 & 0.2609 & 5.72 & 0.2841 & 7.14 & 0.4116 & 5.08 & 0.5335 & 4.91 & 0.4555 & 5.80 \\
1.82 & 0.2985 & 3.35 & 0.3446 & 4.56 & 0.3724 & 5.87 & 0.5313 & 4.12 & 0.6665 & 3.988 & 0.5777 & 4.89 \\
1.96 & 0.3757 & 2.66 & 0.4284 & 3.83 & 0.459 & 5.06 & 0.6391 & 3.48 & 0.7734 & 3.44 & 0.6835 & 4.31 \\
4 & 0.9074 & 1.10 & 0.9302 & 2.11 & 0.9401 & 3.12 & 0.9941 & 2.08 & 0.9995 & 2.08 & 0.9958 & 3.06 \\
\hline
\end{tabular}


Table 5. $P_{1}$ and $A R L_{1}$ of $S_{L}^{2}$ control chart at $n=5, \alpha=0.0027, A R L_{0}=370$, $k / k$ and $\delta_{4}$

\begin{tabular}{lllllllllllll}
\hline \multicolumn{10}{c}{$3 / 3$} & \multicolumn{10}{c}{$S_{L}^{2}$} \\
\hline & $1 / 1$ & & $2 / 2$ & & $3 / 3$ & & $2 / 3$ & & $2 / 4$ & & $3 / 4$ \\
$n_{4}$ & $P_{1}$ & $A R L_{1}$ & $P_{1}$ & $A R L_{1}$ & $P_{1}$ & $A R L_{1}$ & $P_{1}$ & $A R L_{1}$ & $P_{1}$ & $A R L_{1}$ & $P_{1}$ & $A R L_{1}$ \\
\hline 0.04 & 1 & 7 & 1 & 1 & 1 & 3 & 1 & 2 & 1 & 2 & 1 & 3 \\
0.36 & 0.1158 & 8.63 & 0.5922 & 2.94 & 0.8862 & 3.21 & 0.66 & 3.11 & 0.7173 & 3.25 & 0.9442 & 3.21 \\
0.42 & 0.0676 & 14.79 & 0.3642 & 4.31 & 0.6797 & 3.80 & 0.41 & 4.57 & 0.4567 & 4.80 & 0.7674 & 3.73 \\
0.49 & 0.0401 & 24.96 & 0.1995 & 7.06 & 0.4285 & 5.17 & 0.2238 & 7.43 & 0.2494 & 7.79 & 0.4996 & 5.08 \\
0.56 & 0.0242 & 41.27 & 0.1015 & 12.57 & 0.2269 & 8.17 & 0.1115 & 13.04 & 0.1239 & 13.36 & 0.2646 & 7.98 \\
0.64 & 0.015 & 66.75 & 0.0496 & 23.74 & 0.1053 & 14.75 & 0.0531 & 24.55 & 0.0586 & 24.92 & 0.1202 & 14.64 \\
0.72 & 0.0095 & 105.62 & 0.0238 & 46.47 & 0.0447 & 29.78 & 0.0249 & 47.61 & 0.0272 & 46.70 & 0.0494 & 29.39 \\
0.81 & 0.0061 & 163.65 & 0.0114 & 92.65 & 0.0179 & 65.52 & 0.0116 & 93.81 & 0.0126 & 93.50 & 0.0192 & 64.05 \\
0.90 & 0.004 & 248.51 & 0.0055 & 185.72 & 0.007 & 152.93 & 0.0055 & 186.30 & 0.0059 & 187.79 & 0.0072 & 152.44 \\
1 & 0.0027 & 370.37 & 0.0027 & 370.76 & 0.0027 & 370.02 & 0.0026 & 377.00 & 0.0028 & 368.91 & 0.0027 & 371.57 \\
\hline
\end{tabular}

Table 6. $P_{1}$ and $A R L_{1}$ of $S_{U}$ control chart at $n=5, \alpha=0.0027, A R L_{0}=370$, $k / k, k / k+r$ and $\delta_{3}$

\begin{tabular}{|c|c|c|c|c|c|c|c|c|c|c|c|c|}
\hline \multicolumn{13}{|c|}{$S_{U}$} \\
\hline \multirow[b]{2}{*}{$\delta_{3}$} & \multicolumn{2}{|l|}{$1 / 1$} & \multicolumn{2}{|l|}{$2 / 2$} & \multicolumn{2}{|l|}{$3 / 3$} & \multicolumn{2}{|l|}{$2 / 3$} & \multicolumn{2}{|l|}{$2 / 4$} & \multicolumn{2}{|l|}{$3 / 4$} \\
\hline & $p_{1}$ & $A R L_{1}$ & $p_{1}$ & $A R L_{1}$ & $p_{1}$ & $A R L_{1}$ & $p_{1}$ & $A R L_{1}$ & $p_{1}$ & $A R L_{1}$ & $p_{1}$ & $A R L_{1}$ \\
\hline 1 & 0.0027 & 370.37 & 0.0027 & 370.76 & 0.0027 & 370.03 & 0.0026 & 373.71 & 0.0028 & 368.81 & 0.0027 & 368.77 \\
\hline 1.05 & 0.0053 & 189.71 & 0.0055 & 186.51 & 0.0057 & 187.42 & 0.006 & 178.51 & 0.0068 & 171.72 & 0.0064 & 174.27 \\
\hline 1.1 & 0.0094 & 106.93 & 0.0101 & 104.6 & 0.0106 & 106.43 & 0.0121 & 97.71 & 0.0144 & 90.02 & 0.0132 & 93.83 \\
\hline 1.15 & 0.0153 & 65.22 & 0.017 & 64.13 & 0.0182 & 66.31 & 0.0219 & 58.28 & 0.027 & 54.83 & 0.0245 & 55.96 \\
\hline 1.2 & 0.0235 & 42.49 & 0.0267 & 42.32 & 0.0288 & 44.57 & 0.0363 & 37.09 & 0.0462 & 34.82 & 0.0412 & 36.70 \\
\hline 1.25 & 0.0342 & 29.25 & 0.0393 & 29.68 & 0.0428 & 31.87 & 0.056 & 25.72 & 0.0729 & 24.12 & 0.064 & 26.12 \\
\hline 1.3 & 0.0474 & 21.09 & 0.055 & 21.89 & 0.0602 & 23.98 & 0.0814 & 19.12 & 0.1075 & 17.64 & 0.0933 & 19.00 \\
\hline 1.35 & 0.0632 & 15.82 & 0.0739 & 16.85 & 0.0811 & 18.81 & 0.1123 & 14.67 & 0.1498 & 13.64 & 0.1288 & 15.26 \\
\hline 1.4 & 0.0815 & 12.27 & 0.0956 & 13.43 & 0.1051 & 15.28 & 0.1482 & 11.62 & 0.1987 & 10.93 & 0.1696 & 12.32 \\
\hline 2 & 0.3976 & 2.52 & 0.4515 & 3.68 & 0.4828 & 4.89 & 0.6667 & 3.36 & 0.7986 & 3.31 & 0.7099 & 4.17 \\
\hline
\end{tabular}

Table 7. $P_{1}$ and $A R L_{1}$ of $S_{L}$ control chart at $n=5, \alpha=0.0027, A R L_{0}=370$, $k / k, k / k+r$ and $\delta_{4}$

\begin{tabular}{lllllllllllll}
\hline \multicolumn{10}{c}{$2 / 2$} & \multicolumn{10}{c}{$S_{L}$} \\
\hline & $1 / 1$ & & $2 / 3$ & & $2 / 3$ & & $2 / 4$ & $3 / 4$ \\
${$\cline { 2 - 7 }$} }$ & $P_{1}$ & $A R L_{1}$ & $P_{1}$ & $A R L_{1}$ & $P_{1}$ & $A R L_{1}$ & $P_{1}$ & $A R L_{1}$ & $P_{1}$ & $A R L_{1}$ & $P_{1}$ & $A R L_{1}$ \\
\hline 0.2 & 0.5616 & 1.78 & 0.9977 & 2 & 1 & 3 & 0.9997 & 2.00 & 0.9999 & 2.016 & 1 & 3.00 \\
0.6 & 0.0191 & 52.43 & 0.0716 & 17.09 & 0.1577 & 10.76 & 0.0777 & 17.48 & 0.086 & 17.67 & 0.1824 & 10.75 \\
0.65 & 0.0141 & 70.76 & 0.0453 & 25.78 & 0.0951 & 16.01 & 0.0483 & 27.41 & 0.0533 & 26.43 & 0.1081 & 15.43 \\
0.7 & 0.0107 & 93.65 & 0.029 & 38.76 & 0.0566 & 24.46 & 0.0305 & 39.11 & 0.0334 & 40.06 & 0.0631 & 24.44 \\
0.75 & 0.0082 & 121.81 & 0.0188 & 57.9 & 0.0335 & 38.05 & 0.0195 & 59.30 & 0.0212 & 59.07 & 0.0366 & 36.74 \\
0.8 & 0.0064 & 156.02 & 0.0124 & 85.76 & 0.0199 & 59.81 & 0.0127 & 87.37 & 0.0137 & 86.16 & 0.0213 & 58.85 \\
0.85 & 0.0051 & 197.07 & 0.0083 & 125.74 & 0.0119 & 94.52 & 0.0084 & 127.79 & 0.009 & 127.99 & 0.0125 & 93.10 \\
0.9 & 0.0041 & 245.86 & 0.0056 & 182.36 & 0.0072 & 149.48 & 0.0056 & 185.15 & 0.006 & 184.65 & 0.0074 & 148.47 \\
0.95 & 0.0033 & 303.3 & 0.0039 & 261.5 & 0.0044 & 235.78 & 0.0038 & 264.10 & 0.0041 & 264.93 & 0.0044 & 237.43 \\
1 & 0.0027 & 370.37 & 0.0027 & 370.76 & 0.0027 & 370.03 & 0.0026 & 373.48 & 0.0028 & 372.11 & 0.0027 & 367.01 \\
\hline
\end{tabular}


Table 8. $P_{1}$ and $A R L_{1}$ of $|S|_{U}$ control chart at $n=5, \alpha=0.0027, A R L_{0}=370$, $k / k, k / k+r$ and $d^{*}$

\begin{tabular}{|c|c|c|c|c|c|c|c|c|c|c|c|c|}
\hline \multicolumn{13}{|c|}{$|S|_{U}$} \\
\hline \multirow[b]{2}{*}{$d^{*}$} & \multicolumn{2}{|l|}{$1 / 1$} & \multicolumn{2}{|l|}{$2 / 2$} & \multicolumn{2}{|l|}{$3 / 3$} & \multicolumn{2}{|l|}{$2 / 3$} & \multicolumn{2}{|l|}{$2 / 4$} & \multicolumn{2}{|l|}{$3 / 4$} \\
\hline & $P_{1}$ & $A R L_{1}$ & $P_{1}$ & $A R L_{1}$ & $P_{1}$ & $A R L_{1}$ & $P_{1}$ & $A R L_{1}$ & $P_{1}$ & $A R L_{1}$ & $P_{1}$ & $A R L_{1}$ \\
\hline 1 & 0.0027 & 370.37 & 0.0027 & 370.76 & 0.0027 & 370.03 & 0.0026 & 370 & 0.0028 & 370 & 0.0027 & 370 \\
\hline 1.05 & 0.0040 & 250.79 & 0.0042 & 244.81 & 0.0043 & 243.16 & 0.0043 & 238.69 & 0.0048 & 239.53 & 0.0046 & 234.54 \\
\hline 1.1 & 0.0057 & 176.56 & 0.0061 & 169.22 & 0.0064 & 167.88 & 0.0067 & 161.21 & 0.0076 & 155.46 & 0.0073 & 156.97 \\
\hline 1.15 & 0.0078 & 128.55 & 0.0086 & 121.66 & 0.0092 & 120.91 & 0.01 & 116 & 0.0116 & 107.89 & 0.0111 & 109.15 \\
\hline 1.2 & 0.0104 & 96.37 & 0.0118 & 90.51 & 0.0128 & 90.29 & 0.0142 & 84.36 & 0.017 & 79.37 & 0.0161 & 79.05 \\
\hline 1.25 & 0.0135 & 74.130 & 0.0157 & 69.36 & 0.0172 & 69.58 & 0.0196 & 62.81 & 0.0238 & 58.89 & 0.0225 & 60.14 \\
\hline 1.3 & 0.0171 & 58.31 & 0.0203 & 54.55 & 0.0225 & 55.1 & 0.0262 & 49.84 & 0.0324 & 46.04 & 0.0305 & 47.18 \\
\hline 1.35 & 0.0214 & 46.8 & 0.0256 & 43.88 & 0.0287 & 44.67 & 0.0341 & 38.97 & 0.0429 & 37.42 & 0.0401 & 37.07 \\
\hline 1.4 & 0.0262 & 38.23 & 0.0318 & 36.02 & 0.0358 & 36.98 & 0.0434 & 32.20 & 0.0552 & 30.25 & 0.0514 & 30.76 \\
\hline 2 & 0.1234 & 8.11 & 0.1572 & 8.74 & 0.1805 & 10.01 & 0.2447 & 7.67 & 0.3238 & 7.40 & 0.2889 & 8.23 \\
\hline
\end{tabular}

Table 9. $P_{1}$ and $A R L_{1}$ of $|S|_{L}$ control chart at $n=5, \alpha=0.0027, A R L_{0}=370$, $k / k, k / k+r$ and $d^{*}$

\begin{tabular}{|c|c|c|c|c|c|c|c|c|c|c|c|c|}
\hline \multicolumn{13}{|c|}{$|S|_{L}$} \\
\hline \multirow[b]{2}{*}{$d^{*}$} & \multicolumn{2}{|l|}{$1 / 1$} & \multicolumn{2}{|l|}{$2 / 2$} & \multicolumn{2}{|l|}{$3 / 3$} & \multicolumn{2}{|l|}{$2 / 3$} & \multicolumn{2}{|l|}{$2 / 4$} & \multicolumn{2}{|l|}{$3 / 4$} \\
\hline & $P_{1}$ & $A R L_{1}$ & $P_{1}$ & $A R L_{1}$ & $P_{1}$ & $A R L_{1}$ & $P_{1}$ & $A R L_{1}$ & $P_{1}$ & $A R L_{1}$ & $P_{1}$ & $A R L_{1}$ \\
\hline 0.2 & 0.1553 & 6.44 & 0.6141 & 2.87 & 0.8693 & 3.26 & 0.7119 & 2.93 & 0.7767 & 3.07 & 0.9426 & 3.22 \\
\hline 0.6 & 0.0109 & 91.36 & 0.0265 & 42.13 & 0.0471 & 28.6 & 0.0287 & 43.38 & 0.0319 & 41.87 & 0.0542 & 27.44 \\
\hline 0.65 & 0.0088 & 113.24 & 0.019 & 57.38 & 0.0318 & 39.98 & 0.0202 & 57.79 & 0.0224 & 57.09 & 0.0358 & 38.11 \\
\hline 0.7 & 0.0072 & 138.38 & 0.0139 & 77.26 & 0.0216 & 55.79 & 0.0145 & 79.31 & 0.016 & 77.62 & 0.0239 & 53.49 \\
\hline 0.75 & 0.006 & 167 & 0.0102 & 102.89 & 0.0149 & 77.58 & 0.0106 & 104.58 & 0.0116 & 102.30 & 0.0161 & 76.19 \\
\hline 0.8 & 0.005 & 199. & 0.00 & 135 & 0.0 & 107.36 & 0.00 & 139 & 0.00 & 132.88 & 0.011 & 104.68 \\
\hline 0.85 & 0.0042 & 235.62 & 0.0058 & 176.81 & 0.0073 & 147.72 & 0.0059 & 174.74 & 0.0063 & 172.15 & 0.0076 & 146.35 \\
\hline 0.9 & 0.0036 & 276.07 & 0.0045 & 228.36 & 0.0052 & 201.98 & 0.0044 & 232.46 & 0.0048 & 228.26 & 0.0053 & 194.40 \\
\hline 0.95 & 0.0031 & 320.91 & 0.0035 & 292.25 & 0.0037 & 274.33 & 0.0034 & 294.21 & 0.0037 & 286.50 & 0.0038 & 278.27 \\
\hline 1 & 0.0027 & 370.37 & 0.0027 & 370.76 & 0.0027 & 370.03 & 0.0026 & 374.14 & 0.0028 & 369.12 & 0.0027 & 370.25 \\
\hline
\end{tabular}

\section{3. $T_{U}^{2}$ control chart}

The procedure for computing the $P_{1}$ and $A R L_{1}$ of $T_{U}^{2}$ control chart is similar to $|S|_{U}$ and $|S|_{U}$ control charts except difference is at least one elements of $\underline{\mu_{0}}$ is shifted (say $\underline{\mu_{1}}$ ), whereas $\Sigma_{0}$ is stable, that is,

$$
\underline{\mu_{1}}=\left[\begin{array}{l}
\delta_{7} \\
\delta_{8}
\end{array}\right], \delta_{7}=\delta_{8}, \text { and } \Sigma_{0}=\left[\begin{array}{cc}
\sigma_{10}^{2} & \rho \sigma_{10} \sigma_{20} \\
\rho \sigma_{10} \sigma_{20} & \sigma_{20}^{2}
\end{array}\right]
$$

where $\delta_{7} \in \Re$ and $\delta_{8} \in \Re$ represent amount of shift in the in-control process means, thats is, $\mu_{10}$ and $\mu_{20}$ respectively. After that, generate random sample from bivariate normal distribution with $\mu_{0}$ and $\Sigma_{1}$ followed by calculating the $T_{j}^{2}$ and comparing with $U_{T^{2}}$ to decide whether the process is in-control or out-of-control. Rest of the steps for computing $P_{1}$ and $A R L_{1}$ of $T_{U}^{2}$ control chart are similar to $\bar{X}_{U}$ control chart. It is valuable to mention that detection ability of $T_{U}^{2}$ control chart is dependent on the Mahalanobis distance $d$, that is,

$$
d=\sqrt{\left(\underline{\mu_{1}}-\underline{\mu_{0}}\right)^{t} \Sigma_{0}^{-1}\left(\underline{\mu_{0}}-\underline{\mu_{1}}\right)},
$$

and $n$ in respective of the choice of other quantities $\left(\underline{\mu_{1}}\right.$, and $\left.\Sigma_{0}\right)$. This property is termed as directional invariance [14]. Therefore, we have considered shift in form of $d$ as can be seen in many existing studies such as Mehmood et al. [7] and Pignatillo and Runger [14]. Also, for $\alpha=0.0027, A R L_{0}=370$, and some choices of $n, d, k / k$ and $k / k+r$, we have provided $\alpha_{a c t}, A R L_{a c t}, P_{1}$ and $A R L_{1}$ in Table 10. Similarly one may proceed for other 
choices of $\alpha, A R L_{0}, n, d, k / k$ and $k / k+r$. Furthermore, results are described in following points:

- The $\alpha_{a c t}$ and $A R L_{1}$ of $T_{U}^{2}$ control chart are determined equal to $\alpha$ and $A R L_{0}$ (i.e. $\alpha_{a c t}$ $=\alpha=0.0027$ and $\left.A R L_{a c t}=A R L_{0}=370\right)$, respectively for classical and additional runs rules (see Table 1 ).

- The detection ability of FAR based $T_{U}^{2}$ control chart is uniformly outstanding at various choices of $d$ when additional runs rules are plugged relative to classical runs rule in general. In comparison, ARL based $T_{U}^{2}$ control chart is noted superior for small-to-moderate $d$ when additional runs rules are integrated relative to classical rule (see Table 10).

- The $n$ and $d$ are associated with the detection ability of $T_{U}^{2}$ control chart. It is summarized as detection ability of $T_{U}^{2}$ control chart in terms of $P_{1}$ and $A R L_{1}$ increases as size of $n$ and/or $d$ increases (see Table 10).

- The $2 / 4$ runs rule is performed superb with $T_{U}^{2}$ control chart for detection of smallto-moderate $d$ relative to the other runs rules schemes. Also, performance order of various runs rules is as follows: $2 / 4$ is ranked at 1 st position followed by $3 / 4$ at 2 nd, $2 / 3$ at 3 rd, $3 / 3$ at 4 th, $2 / 2$ at 5 th, and $1 / 1$ at last.

Table 10. $P_{1}$ and $A R L_{1}$ of $T_{U}^{2}$ control chart at $n=5, \alpha=0.0027, A R L_{0}=370$, $k / k, k / k+r$ and $d$.

\begin{tabular}{lllllllllllll}
\hline & \multicolumn{10}{c}{$T_{U}^{2}$} \\
\hline & $1 / 1$ & & $2 / 2$ & & $3 / 3$ & & $2 / 3$ & & $2 / 4$ & & $3 / 4$ \\
\cline { 2 - 14 }$y$ & $P_{1}$ & $A R L_{1}$ & $P_{1}$ & $A R L_{1}$ & $P_{1}$ & $A R L_{1}$ & $P_{1}$ & $A R L_{1}$ & $P_{1}$ & $A R L_{1}$ & $P_{1}$ & $A R L_{1}$ \\
\hline 0 & 0.0027 & 370.37 & 0.0027 & 370.76 & 0.0027 & 370.03 & 0.0026 & 374.76 & 0.0028 & 367.76 & 0.0027 & 367.97 \\
0.4 & 0.0094 & 106.89 & 0.011 & 97.04 & 0.0119 & 96.42 & 0.0173 & 72.709 & 0.0208 & 67.74 & 0.0198 & 69.14 \\
0.5 & 0.0149 & 67.11 & 0.019 & 57.89 & 0.0215 & 57.15 & 0.0332 & 41.84 & 0.0411 & 37.78 & 0.0403 & 38.57 \\
0.6 & 0.0236 & 42.34 & 0.0327 & 35.04 & 0.0387 & 34.54 & 0.0614 & 24.60 & 0.0778 & 22.67 & 0.0781 & 22.74 \\
0.8 & 0.056 & 17.85 & 0.0884 & 14.35 & 0.1113 & 14.52 & 0.1756 & 9.74 & 0.2265 & 9.26 & 0.2324 & 9.31 \\
0.9 & 0.0829 & 12.06 & 0.1362 & 9.86 & 0.1739 & 10.25 & 0.2682 & 6.85 & 0.3434 & 6.72 & 0.352 & 6.76 \\
1 & 0.1191 & 8.4 & 0.1999 & 7.12 & 0.2558 & 7.67 & 0.3817 & 5.9 & 0.4802 & 4.10 & 0.4893 & 5.56 \\
1.1 & 0.1657 & 6.03 & 0.2792 & 5.4 & 0.3542 & 6.05 & 0.5076 & 4.99 & 0.6214 & 3.15 & 0.628 & 4.41 \\
1.2 & 0.2233 & 4.48 & 0.3714 & 4.29 & 0.4627 & 5.01 & 0.6335 & 3.78 & 0.7494 & 2.1 & 0.7515 & 3.72 \\
1.4 & 0.3681 & 2.72 & 0.5728 & 3.05 & 0.6767 & 3.86 & 0.8383 & 2.8 & 0.9206 & 2 & 0.9161 & 2.15 \\
\hline
\end{tabular}

\section{Real life example}

In this section, we conduct a comparative analysis between FAR and ARL based control charts with runs rules by using the practical data sets. The purpose of comparative analysis with aid of practical data sets is to know whether the behavior of FAR and ARL based control charts remains similar as described in Section 3 using the statistical performance indicators. To achieve the purpose, we consider a data set from Alwan [1] which refers back to [17] containing the data on 204 consecutive measurement on the electrical resistance of insulation in megohms. The data set is normally distributed with mean $=4498.076$ and the standard deviation $=328$. Afterwards, we have developed a code in $\mathrm{R}$ language to implement the FAR and ARL based $\bar{X}_{U}$ control charts for $k / k=1 / 1,2 / 2,3 / 3, \alpha=0.0027$, and $A R L_{0}=370$ (see Figures 1-2).

The FAR based $\bar{X}_{U}$ control chart shows 3,5 and 6 out-of-control signals for the $1 / 1$, $2 / 2$ and $3 / 3$ runs rules, respectively (see Figure 1 ). It is worthy to mention that numbers of out-of-control signals given by ARL based $\bar{X}_{U}$ control chart with varying choices of runs rules are equal to the case of FAR based $\bar{X}_{U}$ control chart (see Figure 2). This indicates that behavior of FAR and ARL based $\bar{X}_{U}$ control charts are identical. Also, additional runs rules contributes towards detection of small and moderate variations. This comparative 
discussion is in accordance with the statistical results provided in Section 3.1. On the similar lines, one may attempt for the other choices of control charts.

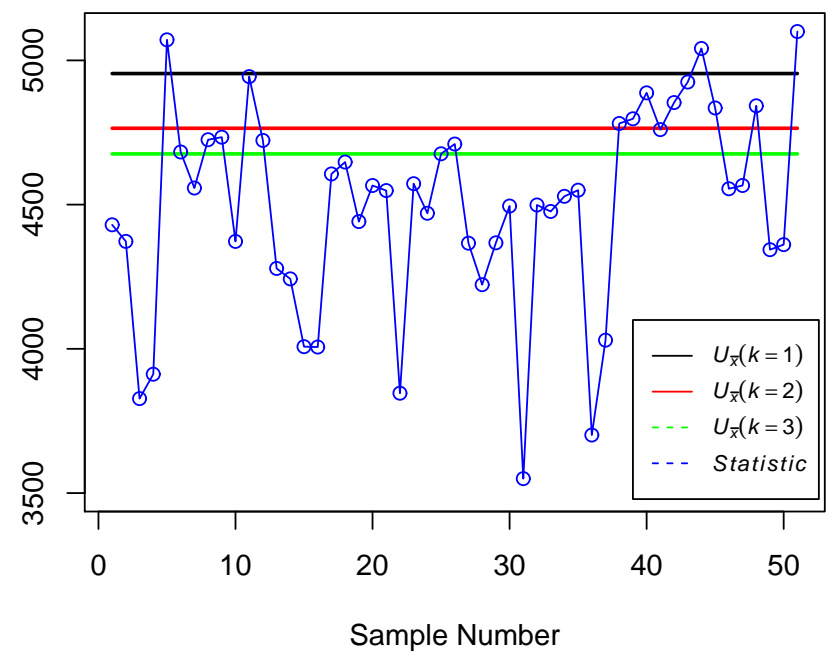

Figure 1. FAR based $\bar{X}_{U}$ control chart for varying choices of runs rules $(k=$ $1,2,3)$ and $\alpha=0.0027$

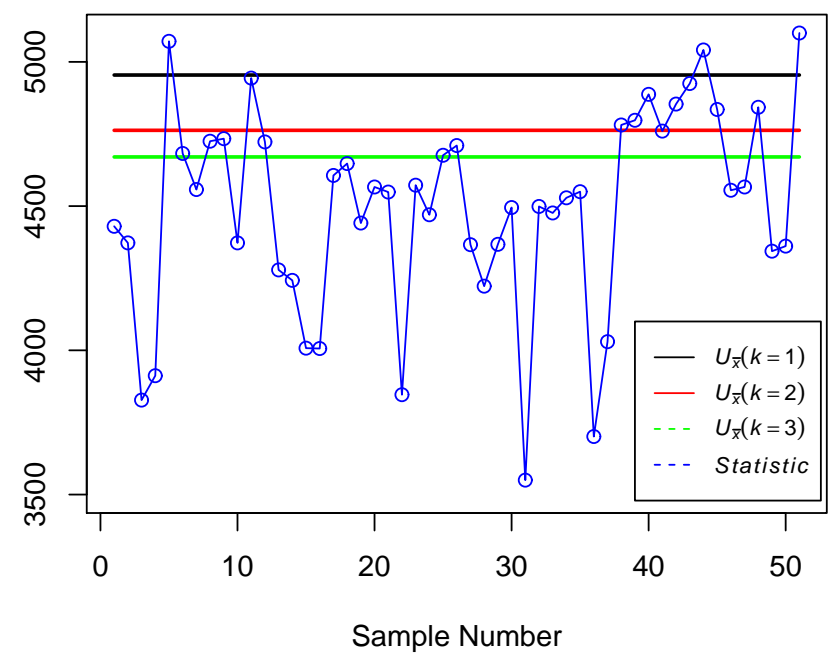

Figure 2. ARL based $\bar{X}_{U}$ control chart for varying choices of runs rules $(k=$ $1,2,3)$ and $A R L_{0}=370$

\section{Summary, conclusions and future recommendations}

In this article, we have described comparative behavior of false alarm rate (FAR) and average run length (ARL) based control charts with runs rules. In the list of univariate 
and multivariate control charts, we have included upper-sided and lower-sided mean, variance, standard deviation, generalized variance, and Hotelling's. For comparative analysis and discussions, we have included actual false alarm rate, power, in-control actual average run length, and out-of-control average run length as performance measures. Furthermore, performance measures are computed by using Monte Carlo simulation procedures as computation methodology. Besides, diverse results are presented by taking into account numbers of factors. The detection ability of FAR based lower-sided and upper-sided control charts are remained uniformly higher when additional runs rule are incorporated relative to classical runs rule. Also, detection ability of ARL based lower-sided control charts are recorded outstanding for small-to-moderate shifts when additional runs rules are employed relative to classical runs rules. In brief, performance order of decision rules with FAR based lower-sided and upper-sided control charts are persistent, whereas performance order of decision rules with ARL based control charts are dependent on the circumstances, that is, sample size, size of shift, class of control chart (location and dispersion), and side of control limit (upper-sided and lower-sided). Lastly, we have provided a real life example using the data on electrical resistance of insulation. In the real life example, we have recorded that behavior of FAR and ARL based control charts using the real data sets are similar to the behavior using the statistical measures.

The scope of current study covers the processes in which characteristics follows normal distribution and parameters are known. It is often that process distribution is non-normal or unknown, and parameters are unknown. Therefore, it would be excellent to conduct an efficient study in future for non-normal distribution and parameters are unknown. Likewise, one may contribute a study by involving robust techniques (e.g. robust estimators and non-parametric) with control charts. An interesting study can be added on the topic of comparative analysis between cumulative sum (CUSUM) and exponentially weighted moving average (EWMA) control charts with runs rules.

Acknowledgment. This study was partially funded by the Ministry of Higher Education, Malaysia, through the UTM Encouragement Research Grant (grant number 19J38).

\section{References}

[1] L.C. Alwan, Statistical Process Analysis, McGraw-Hill International Editions, Singapore, 2000.

[2] S. Chakraborti and S. Eryilmaz, A nonparametric Shewhart-type signed-rank control chart based on runs, Comm. Statist. Simulation Comput. 36 (2), 335-356, 2007.

[3] C.W. Champ and W.H. Woodall, Exact results for Shewhart control charts with supplementary runs rules, Technometrics 29 (4), 393-399, 1987.

[4] M.B. Khoo, Design of runs rules schemes, Qual. Eng. 16 (1), 27-43, 2003.

[5] M. Klein, Two alternatives to the shewhart $x$ control chart, J. Qual. Technol. 32 (4), 427-431, 2000.

[6] J.C. Malela-Majika, S.C. Shongwe and P. Castagliola, One-sided precedence monitoring schemes for unknown shift sizes using generalized 2-of- $(h+1)$ and $w$-of-w improved runs-rules, Comm. Statist. Theory Methods, 1-35, 2020.

[7] R. Mehmood, M.H. Lee, M. Riaz, B. Zaman and I. Ali, Hotelling T ${ }^{2}$ control chart based on bivariate ranked set schemes, Comm. Statist. Simulation Comput., 1-28, 2019.

[8] R. Mehmood, M.S. Qazi and M. Riaz, On the performance of $\bar{X}$ control chart for known and unknown parameters supplemented with runs rules under different probability distributions, J. Stat. Comput. Simul. 88 (4), 675-711, 2018.

[9] R. Mehmood, M. Riaz and R.J.M.M. Does, Control charts for location based on different sampling schemes, J. Appl. Stat. 40 (3), 483-494, 2013. 
[10] R. Mehmood, M. Riaz and R.J.M.M. Does, Efficient power computation for $r$ out of $m$ runs rules schemes, Comput. Statist. 28 (2), 667-681, 2013.

[11] R. Mehmood, M. Riaz and R.J.M.M. Does, Quality quandaries: on the application of different ranked set sampling schemes, Qual. Eng. 26 (3), 370-378, 2014.

[12] R. Mehmood, M. Riaz, T. Mahmood, S.A. Abbasi and N. Abbas, On the extended use of auxiliary information under skewness correction for process monitoring, Trans. Inst. Meas. Control. 39 (6), 883-897, 2017.

[13] D.C. Montgomery, Introduction to Statistical Quality Control, John Wiley Sons, New York, 2009.

[14] Jr, J.J. Pignatiello and G.C. Runger, Comparisons of multivariate cusum charts, J. Qual. Technol. 22 (3), 173-186, 1990.

[15] M. Riaz, R. Mehmood and R.J.M.M. Does, On the performance of different control charting rules, Qual. Reliab. Eng. 27 (8), 1059-1067, 2011.

[16] D.K. Shepherd, S.E. Rigdon and C.W. Champ, Using runs rules to monitor an attribute chart for a markov process, QTQM 9 (4), 383-406, 2012.

[17] W.A. Shewhart, Economic Control of Quality of Manufactured Product, ASQ Quality Press, 1931.

[18] S.C. Shongwe, On the design of nonparametric runs-rules schemes using the markov chain approach, Qual. Reliab. Eng. 36 (5), 1604-1621, 2020.

[19] S.C. Shongwe, J.C. Malela-Majika and T. Molahloe, One-sided runs rules schemes to monitor autocorrelated time series data using a first-order autoregressive model with skip sampling strategies, Qual. Reliab. Eng. 35 (6), 1973-1997, 2019.

[20] S. Shongwe, J.C. Malela-Majika and E. Rapoo, One-sided and two-sided w-of-w runsrules schemes: An overall performance perspective and the unified run-length derivations, J. Probab. Stat., 1-20, 2019.

[21] E.C. Western, Statistical Quality Control Handbook, Western Electric Company, Indianapolis, 1956.

[22] B. Zaman, M. Riaz and S.A. Abbasi, On the efficiency of runs rules schemes for process monitoring, Qual. Reliab. Eng. 32 (2), 663-671, 2016. 